\title{
1 Pharmacokinetics of fluoride in human adults: the effect of exercise.
}

2 Maria Mahmood ${ }^{a}$, Liane B Azevedo ${ }^{\mathrm{b}}$, Anne Maguire ${ }^{\mathrm{c}}$, M Buzalaf ${ }^{\mathrm{d}}$, Fatemeh Vida Zohoori ${ }^{\mathrm{a}}$

$3 \quad{ }^{\text {a }}$ School of Health and Life Sciences, Teesside University, Middlesbrough, UK

$4 \quad{ }^{\mathrm{b}}$ School of Human and Health Sciences, University of Huddersfield, Huddersfield, UK

$5 \quad{ }^{\mathrm{c}}$ School of Dental Sciences, Faculty of Medical Sciences, Newcastle University, Newcastle-upon-

6 Tyne, UK

$7 \quad$ d Bauru Dental School, University of Sao Paulo, Brazil

8

9

10

11 Running title: Effect of exercise on fluoride metabolism

12 Keywords: fluoride, renal excretion, exercise intensity

* Corresponding author

14 Professor FV Zohoori

15 School of Health and Life Sciences, Teesside University

16 Middlesbrough, TS1 3BA, UK

17 Tel: $+44(0) 1642342973$

18 Fax: +44 (0) 1642342770

19 Email: v.zohoori@tees.ac.uk 


\section{Abstract:}

The literature is sparse in terms of the effect of exercise on the pharmacokinetics of fluoride $(\mathrm{F})$ in humans. In a 4-treatment repeated measures cross-over study, we investigated F pharmacokinetics following no exercise (control) and three exercise intensity conditions (light, moderate and vigorous) in healthy adults. At a pre-experimental session, 8 participants (18-30y) residing in a non-fluoridatedarea, underwent a $\mathrm{VO}_{2 \text { max }}$ test to guide the three exercise intensities for the experimental sessions. Participants were on a F-free regime one week before and throughout the four experimental weeks. We measured urinary F excretion (UFE), maximum plasma concentration (Cmax), lag time of Cmax (Tmax), and Area Under the Curve (AUC) for plasma F concentration against time, following F ingestion then no, light, moderate and vigorous exercise. Results showed no statistically significant difference in Tmax among all sessions; whereas Cmax for moderate exercise $(226.2 \mathrm{ng} / \mathrm{ml})$ was significantly higher than for no $(27.0 \mathrm{ng} / \mathrm{ml} ; \mathrm{p}<0.001)$, light $(105.6 \mathrm{ng} / \mathrm{ml} ; \mathrm{p}=0.016)$ and vigorous (94.2ng/ml; $\mathrm{p}=0.008$ ) exercise. Mean AUC over 0 to 90 min following $\mathrm{F}$ ingestion was also significantly higher in moderate exercise than for no $(\mathrm{p}<0.001)$, light $(\mathrm{p}=0.004)$ and vigorous ( $\mathrm{p}=0.001$ ) exercise. Mean UFE over $0-14 \mathrm{~h}$ was $638.8,718.7,574.6$ and $450.5 \mu \mathrm{g}$ for no, light, moderate and vigorous exercise, with no statistically significant differences among different sessions. In conclusion, this human experimental study suggests that moderate exercise may increase the fraction of $\mathrm{F}$ absorbed systemically which is therefore available to produce a biological effect. Future studies should be conducted with larger samples, different age groups and using different $\mathrm{F}$ doses. 
While the effectiveness of topical exposure to fluoride $(\mathrm{F})$ in the prevention of dental caries has been well demonstrated, excessive exposure to systemic F can have some health side effects including dental and skeletal fluorosis (ten Cate and Buzalaf, 2019). Undesirable health effects of F can be related not only to the body's total $\mathrm{F}$ intake but, more importantly, to the extent of $\mathrm{F}$ retention in the body. Genetic and environmental factors such as stage of skeletal development, acid-base balance and exercise have been suggested to influence metabolism and body retention of $\mathrm{F}$ (Buzalaf and Whitford, 2011; Buzalaf, 2018). Understanding F metabolism and its physiological characterisation is therefore very important if we are to avoid or minimise side effects of systemic F exposure.

The pharmacokinetics of $\mathrm{F}$ is mainly controlled by $\mathrm{pH}$ and storage in bone, because the coefficient of permeability of lipid bilayer membranes to hydrogen fluoride (HF) is a million times higher than to $\mathrm{F}$ ion (Buzalaf and Whitford, 2011). Therefore, factors affecting systemic $\mathrm{pH}$ (in cells, tissues and fluids) could play an important role in the body's absorption, distribution, excretion and retention of F. After absorption, $\mathrm{F}$ concentrations of plasma rise promptly due to the rapid absorption of $\mathrm{F}$ from the stomach and reach their peak within 20-60 min. Plasma F concentration normally returns to preingestion levels during the next few hours depending on the F dose. Plasma F concentrations are not homeostatically controlled and therefore fluctuate according to the F dose, body deposition and excretion. Under normal conditions, almost $60 \%$ of a healthy adult's and $45 \%$ of a healthy child's daily absorbed $\mathrm{F}$ is excreted in urine and most (about 99\%) of the body-retained $\mathrm{F}$ is associated with calcified tissues (Buzalaf and Whitford, 2011).

F has been reported as one of a few known agents that can stimulate osteoblast proliferation (Palmer and Wolfe, 2005). However, different doses of F display a biphasic effect on osteoclast cell viability, differentiation, formation and function: a low $\mathrm{F}$ dose stimulates them, whereas a high dose inhibits them (Yu et al., 2018). Furthermore, a decline in expression of osteocytes but a rise in expression of osteoblasts has been linked to exercise (Schwab and Scalapino, 2011), in particular weight-bearing exercise (Willems et al., 2017). Therefore, the pharmacokinetics of F may be influenced by alterations in physiological responses to acute and chronic exercise. Changes in body $\mathrm{F}$ retention could be 
important in terms of the effect of $\mathrm{F}$ on tooth and bone development and the timing of $\mathrm{F}$ ingestion when fluorides are used in dental caries prevention.

The literature is sparse and contradictory in terms of the effect of exercise on pharmacokinetics of $\mathrm{F}$ in humans. The only human experimental study, comparing F concentration in plasma and urine between exercised and non-exercised groups, reported higher plasma F concentrations with moderate and vigorous intensity exercise as well as a reduction in urinary $\mathrm{F}$ excretion with moderate exercise compared with a non-exercised control in young adults (Zohoori et al., 2015). Conversely, two animal studies have reported a significant reduction in plasma $\mathrm{F}$ concentration in rats exposed to a one-hour treadmill running exercise (Whitford, 1996; Lombarte et al., 2013). More recently, an animal study (Amaral et al., 2018) reported no effect of high intensity training exercise on plasma $\mathrm{F}$ in fluorosissusceptible mice.

The aim of this present study was to investigate the F pharmacokinetics following no exercise and three exercise intensity conditions (light, moderate and vigorous) in healthy adults. The objectives were to compare urinary F excretion (UFE) and plasma F concentration among no, light, moderate and vigorous exercise intensities.

\section{Methods:}

\subsection{Participants}

This study was conducted according to the guidelines laid down in the Declaration of Helsinki and all procedures involving human participants were approved by the Research Governance and Ethics Committee, School of Health and Social Care, Teesside University (Protocol number 066/15). Prior to the experiment, all participants provided written informed consent.

The target sample size for this exploratory study was eight participants, based on the only human study by Zohoori et al (n=9) (Zohoori et al., 2015) as well as the animal studies by Whitford ( $\mathrm{n}=8$ ) (Whitford et al., 1988), Lombarte et al $(\mathrm{n}=10)$ (Lombarte et al., 2013) and Amaral et al $(\mathrm{n}=8)$ (Amaral et al., 2018). 
The study participants were healthy adult volunteers, from both genders; aged between 18 and 35 years; weighing over $50 \mathrm{~kg}$; with no history of acid-base disturbance and not receiving a therapeutic diet. Participants had to be considered at least moderately active according to the International Physical Activity Questionnaire (i.e. 5 or more days of moderate-intensity activity of at least $30 \mathrm{~min}$ per day) (Craig et al., 2003) and "ready" to engage in the prescribed exercise according to the Physical Activity Readiness Questionnaire (American College of Sports Medicine, 2007). All participants were residing in a non-fluoridated area, with a water $\mathrm{F}$ concentration of $<0.3 \mathrm{ppm}$.

\subsection{Experimental Design}

This experiment was designed as a four-treatment repeated measures cross-over study, comparing observations within individuals.

\section{Pre-experimental Procedures}

Participants were invited to attend a pre-experimental session, when height and weight was measured using a stadiometer (Leicester Height Measure, Child Growth Foundation, London, United Kingdom) and a calibrated scale (Seca 220, Seca Weighing and Measuring Systems, Germany). Each individual participant underwent a $\mathrm{VO}_{2 \text { max }}$ test, performed following a standard cycle ergometer protocol (Evans and White, 2009) to determine the exercise intensities (light, moderate and vigorous) for the experimental sessions. Following a 5-minute warm up at 25 Watts, the test started at 25 Watts with the intensity being increased by 25 Watts every 2 minutes until exhaustion. Cycle ergometer power output (PowerTap Cycleops400, USA) and participant's Rate of Perceived Exertion (RPE) using the CR-10 RPE scale (Noble et al., 1983) were recorded every minute. Heart rate was monitored throughout the exercise sessions with a heart rate monitor (Polar RS400, Polar Electro Oy, Finland) and recorded at every minute during exercise. The three exercise intensities (light, moderate and vigorous) were determined by using the v-slope method (Beaver et al., 1986) and defined for each participant as: (i) light intensity: one load below Ventilatory Threshold (VT) 1; (ii) moderate intensity: the load at VT and; (iii) vigorous intensity: one load above VT. 
In order to minimise $\mathrm{F}$ exposure from all other sources during the study, participants were placed on a

121 F-free regime one week before, as well as during, the whole experimental period. They were provided

122

123

124

125

126

127

130

131

132

with a F-free toothpaste to use and instructed to avoid drinking tea, beer and tap-water (if leaving their stated residential area) and eating seafood during the washout and experimental periods (i.e. for a total of five consecutive weeks). Participants were asked to refrain from performing exercise, other than habitual walking, for $48 \mathrm{~h}$ prior to and during experimental sessions.

\section{Experimental sessions}

After the one-week washout period, each participant underwent four randomly allocated experimental sessions; one no-exercise (control) session and three exercise sessions at different intensities (light, moderate and vigorous) with approximately a week's gap between sessions (Figure 1).

The study was conducted in an exercise laboratory at the same time of day for all experimental sessions to control for circadian rhythms.

Participants attended the laboratory at around 9:00 am on each experiment day, having fasted overnight. A venous cannula was inserted into the antecubital fossa of each participant's arm, by an experienced nurse, for intravenous blood samples collection (as explained in the next section). A baseline venous blood sample $(5 \mathrm{ml})$ was collected from each participant before they were provided with a low-F breakfast $(<10 \mu \mathrm{gF})$ which comprised a cereal bar, a banana and fruit juice. In order to control the influence of $\mathrm{F}$ from diet during the experimental sessions, the same standardised low $\mathrm{F}$ breakfast was consumed by all participants at approximately the same time in each of their four sessions.

After breakfast, all participants (in both control and exercise sessions) were given a $1 \mathrm{mgF}$ tablet (Endekay Fluotabs 2.2mg NaF, Manx Pharma Ltd, Warwick, UK) to ingest. Participants then either rested (control session) or undertook the exercise (exercise sessions) at approximately 9:30am. Participants from the exercise group were fitted with a heart rate monitor belt (Polar RS400, Polar Electro Oy, Finland). Participants warmed up for 5 minutes at a self-selected speed before initiating the designated exercise intensity on the cycle ergometer for 20 minutes. 
Urine samples

148 Pooled urine samples were collected by spontaneous voiding over a $24 \mathrm{~h}$ cycle during four time periods: 1) A nocturnal sample collected from midnight before the experimental (control or exercise) session up until about 09.00am (Baseline, pre-F tablet/pre-exercise); 2) A ‘09.00am to 12.00pm’ sample during the experimental session ( $0-3 \mathrm{~h}$ post-F tablet ingestion); 3) A ' $12.00 \mathrm{pm}$ to $17.00 \mathrm{pm}$ ' sample during the experimental session ( $3-8 \mathrm{~h}$ post-F tablet ingestion) and; 4) A ' $17.00 \mathrm{pm}$ through to just before bed-time' sample ( 23.00pm) on the experimental day (8-14h post-F tablet ingestion).

Blood plasma:

A $5 \mathrm{ml}$ venous blood sample was collected after overnight fasting, prior to taking breakfast (Baseline, T0). An additional four blood samples ( $5 \mathrm{ml} / \mathrm{sample})$ were then collected at 30, 45, 60 and 90 minutes after ingestion of the F-tablet, providing samples $\mathrm{T} 1$ to $\mathrm{T} 4$.

\subsection{Analytical Procedure}

F concentration $(\mu \mathrm{g} / \mathrm{ml})$ of urine samples was measured directly after adding total ionic strength adjustment buffer III (Orion Research) to standards and samples, using a F-ion-selective electrode (FISE, Model Orion 9609BNWP, Thermo Scientific, USA) coupled to a potentiometer (Model 720A+). F concentrations in plasma $(\mathrm{ng} / \mathrm{ml})$ and breakfast items $(\mu \mathrm{g} / \mathrm{g})$ were measured, in triplicate, by a hexamethyldisiloxane (HMDS)-facilitated diffusion method (Taves, 1968) which has been previously reported in detail (Martínez-Mier et al., 2011). In summary, $1 \mathrm{ml} \mathrm{H}_{2} \mathrm{SO}_{4}$ saturated with HMDS was added to $1 \mathrm{ml}$ of sample (and standards) in a petri-dish and left at room temperature to diffuse overnight. An alkaline solution $(50 \mu \mathrm{l}$ of $\mathrm{NaOH}(0.05 \mathrm{~N})$, placed as 5 drops on the inside of the dish lid), was used to trap the released $\mathrm{F}$. After a minimum of $16 \mathrm{~h}$ diffusion, the $\mathrm{NaOH}$ drops were combined as a single drop and $20 \mu \mathrm{l}$ acetic acid $(0.20 \mathrm{~N})$ added. The F-ISE electrode was then placed in contact with the combined solution and the $\mathrm{mV}$ reading recorded. A calibration curve was used to calculate F concentration of the sample. 
171 The reliability of the methods used was specifically confirmed by re-analysis of a minimum $10 \%$ of

172 samples. All sample analysis and re-analysis was conducted in triplicate.

$173 \quad$ 1.2.5 Data handling and analysis

174 Urine:

175 Urinary F excretion (UFE) in each individual time-controlled urine sample was calculated by

176 multiplying the $\mathrm{F}$ concentration $(\mu \mathrm{g} / \mathrm{ml})$ of the urine sample by its corresponding volume $(\mathrm{ml})$.

177 Baseline-adjusted UFE was calculated by subtracting the baseline UFE from the UFE of each sample.

178 The sums of the amount of $\mathrm{F}$ excreted in urine for the periods during and after each experimental

179 session for each participant were used to calculate the total post-F tablet UFE (Periods 2-4 inclusive:

180 representing a $14 \mathrm{~h}$ period).

181 The UFE rate $(\mu \mathrm{g} / \mathrm{h})$ for each given time period was calculated by dividing UFE for each time period by the duration of the corresponding collection period (h).

183 Overall relative UFE (\%) was calculated by dividing the baseline-adjusted UFE ( $\mu \mathrm{g})$ for a given time 184 period by the ingested $\mathrm{F}$ dose (i.e. $1 \mathrm{mg}=1000 \mu \mathrm{g}$ ) multiplied by 100 .

Plasma:

Baseline-adjusted plasma $\mathrm{F}$ concentration ( $\mathrm{ngF} / \mathrm{ml}$ ) was calculated by subtracting the baseline plasma $\mathrm{F}$ concentration from the $\mathrm{F}$ concentration in each plasma sample.

Maximum F concentration ( $\mathrm{Cmax}$ ) was calculated using the mean maximum baseline-adjusted plasma F concentration following F dose. Lag time to maximum F concentration (Tmax) was estimated using graphs plotting plasma F concentration against time. Area under the curve (AUC) (ng/min/ml) was calculated using the following equation:

$192 \mathrm{AUC}=\sum_{i=0}^{n-1} 0.5\left(c_{i}+c_{i+1}\right)\left(t_{i+1}-t_{i}\right)$, where:

193 ( $\mathrm{t}$ ) is the number of minutes after $\mathrm{F}$ dose - the first time point is time 0 and $(\mathrm{Ci})$ is the value of $\mathrm{C}$ at 194 time $t_{\mathrm{i}}$. 
2.6 Statistical Analysis

197 Descriptive data are presented and statistically significant differences among groups were initially detected using repeated measures ANOVA and further investigated using a post-hoc test (Tukey). Statistical significance was set at $\alpha<0.05$ and all analysis performed using SPSS version 22 .

\section{Results:}

All those invited participated and eight participants (4 males and 4 females) took part in the study. The mean (SD) age, height, weight and BMI for females were: $23.7(7.2)$ years, $165.5(3.5) \mathrm{cm}, 64.2$ (2.5) $\mathrm{kg}$ and $23.5(1.6) \mathrm{kg} / \mathrm{m}^{2}$; and for males were: $25.0(6.0)$ years, $176.2(6.0) \mathrm{cm}, 74.2(9.9) \mathrm{kg}$ and $23.7(2.1) \mathrm{kg} / \mathrm{m}^{2}$, respectively.

3.1 Accuracy of the analytical method

The accuracy of the analytical method was confirmed by comparing the analysis and re-analysis measurements. The results showed no statistically significant differences between the two sets of measurements. The mean (SD) difference for urine samples was $0.009(0.002) \mathrm{mgF} / \mathrm{l}(\mathrm{n}=16)$ and for plasma samples was $0.004(0.001) \mathrm{ngF} / \mathrm{ml}(\mathrm{n}=20)$. (37.5) and 112.5 (37.5) Watts for females and 68.7 (37.0), 106.2 (37.0) and 137.5 (37.5) Watts for males, respectively. Mean (SD) maximum heart rates (HR) were 176.0 (12.6) and 160.7 (25.7) bpm and mean RPEs (Rate of Perceived Exertion) at the end of the $\mathrm{VO}_{2 \max }$ test were $4.7(0.8)$ and 7.7 (1.3)

216 in females and males, respectively.

217 Mean (SD) plasma F concentrations, during the control and exercise sessions, according to the different time periods are presented in Table 1 and the pharmacokinetic variables in Table 2. 

baseline fasting plasma F concentration was 31.80 (26.2) ng/ml. Mean baseline-adjusted plasma F concentrations across the 90 minutes post-F ingestion for all experimental sessions are shown in Figure 2.

All experimental sessions followed a similar trend in plasma F concentration, peaking between 30 to 60 minutes post-F ingestion with a Tmax ranging from 43 min for light exercise to 50 and 51 min for control and vigorous exercise, respectively. The highest Cmax was found for moderate exercise (226.2 $\mathrm{ngF} / \mathrm{ml})$ followed by light $(105.6 \mathrm{ngF} / \mathrm{ml})$, vigorous exercise $(94.2 \mathrm{ngF} / \mathrm{ml})$ and control $(27.0$ $\mathrm{ngF} / \mathrm{ml})$. $\mathrm{AUC}_{(0-90 \mathrm{~min})}$ ranged from $15058 \mathrm{ngF} / \mathrm{min} / \mathrm{ml}$ for moderate exercise to $1474 \mathrm{ngF} / \mathrm{min} / \mathrm{ml}$ for control.

Repeated measures analysis of variation (ANOVA) showed no statistically significant difference in Tmax among all sessions, whereas Cmax for moderate exercise was statistically significantly higher compared to no $(\mathrm{p}<0.001)$, light $(\mathrm{p}=0.016)$ and vigorous $(\mathrm{p}=0.008)$ exercise. $\mathrm{AUC}_{(0-90 \mathrm{~min})}$ was also statistically significant higher at moderate exercise intensity compared to no $(\mathrm{p}<0.001)$, light $(\mathrm{p}=$ $0.004)$ and vigorous $(\mathrm{p}=0.001)$ exercise.

The mean (SD) UFE at baseline was $109.2(100.7) \mu \mathrm{gF}$ for the total of 32 experimental sessions undertaken overall by the eight participants. Mean (SD) UFEs for the different time periods during the control and exercise sessions are presented in Table 3.

No statistically significant differences in UFE were found between the no exercise and three different exercise intensities for any individual time period, nor for total post-F tablet period (i.e. 0 -14h post-F tablet ingestion).

Mean baseline-adjusted UFE rates across the 4 time-controlled periods of urine collection are shown in Figure 3.

Light, moderate and vigorous intensity exercise resulted in lower mean baseline-adjusted UFE rates over the $0-3 \mathrm{~h}$ post-F tablet period (light 41.1 , moderate 25.6 and vigorous $35.3 \mu \mathrm{gF} / \mathrm{h}$,) in comparison with no exercise $(62.6 \mu \mathrm{gF} / \mathrm{h})$; however, the differences were not statistically significant. Furthermore, 
there were no statistically significant differences in baseline-adjusted UFE rates among different exercise intensities (including no exercise) for any individual time period.

Mean overall relative UFE (i.e. proportion of ingested F dose excreted in urine) for each time period was $21 \%$ for $0-3 \mathrm{~h}$ post-F tablet, $20 \%$ for $3-8 \mathrm{~h}$ post-F tablet and $16 \%$ for $8-14 \mathrm{~h}$ post-F tablet, with an overall relative UFE of $59 \%$ for $0-14 \mathrm{~h}$ post-F tablet.

\section{Discussion:}

This study provides the first data on the effects of exercise on F pharmacokinetics in healthy adults. The results suggest that moderate exercise may result in higher $\mathrm{F}$ absorption and consequently higher body F retention. These observations could be particularly important in communities with fluoridation programmes such as school-based milk fluoridation where children consume fluoridated milk just before mid-morning playtime (e.g. in UK school milk fluoridation programmes).

The mean Tmax (50 min) and $\mathrm{AUC}_{(0-90 \mathrm{~min})}(1474 \mathrm{ngF} / \mathrm{min} / \mathrm{ml})$ reported in our study for the control session (no exercise, received $1 \mathrm{mgF}$ tablet) were within the corresponding ranges of 43.1-56.6 min and 752-1562 ngF/min/ml, respectively, reported for 21-35 year old English adults given a F dose of $0.5 \mathrm{mg}$ (500 $\mathrm{ml}$ of fluoridated water containing almost $1 \mathrm{mgF} / \mathrm{L}$ ) (Maguire et al., 2005). However, the mean Cmax (27.0 ng/ml) for the no exercise (control) session in our study was higher than the corresponding range of 9.2-19.0 ng/ml reported for English adults (Maguire et al., 2005). Since F dose is an important factor influencing F pharmacokinetics, the observed higher pharmacokinetic parameters in our study, compared to the study by Maguire et al (2005) could be explained by the larger amounts of $\mathrm{F}$ ingested by participants in our study.

Our study found a non-statistically significant trend for an overall lower UFE with greater exercise intensity. However, the overall mean plasma $\mathrm{F}$ concentrations at different time points, as well as Cmax and $\mathrm{AUC}_{(0-90 \min )}$ were higher for the exercise sessions compared to the no exercise (control) session (Tables 1 and 2; Figure 1). These findings imply that exercise could affect the pharmacokinetics of F, i.e. increasing $\mathrm{F}$ absorption but decreasing $\mathrm{F}$ excretion. However, the 
mechanisms by which exercise could alter $\mathrm{F}$ metabolism remain unclear. The increase in cardiac output and consequently muscle and skeletal blood flow following exercise may lead to an increase in the rate of $\mathrm{F}$ absorption and body distribution to muscles and bones. Additionally, exercise could affect renal clearance of $\mathrm{F}$ from kidneys in two ways: (a) increase the activity of sympathetic nervous system, resulting in vasoconstriction within the kidney which would then reduce renal blood flow and glomerular filtration rate (GFR); and (b) increase production of lactic acid by muscle which would increase the renal reabsorption of $\mathrm{F}$ (Whitford, 1996; Buzalaf and Whitford, 2011). These changes would further lessen the renal excretion of $\mathrm{F}$ but tend to increase levels of $\mathrm{F}$ in plasma.

Our study found no statistically significant difference in Tmax among different intensities of exercise including no exercise (control). However, our study showed that the mean values for Cmax and AUC $_{(0-90 \mathrm{~min})}$ were statistically significantly higher for moderate exercise compared with light and vigorous exercise as well when compared with no exercise (control). A study with nine adults (Zohoori et al., 2015) also reported higher plasma F concentrations, although not statistically significant, for moderate intensity exercise compared with control, light and vigorous exercise. Gastric emptying has been shown to increase with increasing exercise intensities up to $65 \% \mathrm{VO}_{2} \max$ (moderate intensity), but it decreases above an intensity of $75 \% \mathrm{VO}_{2} \max$ (vigorous intensity) (Neufer, 1989). Cardiac output following an increased work rate increases in an almost linear manner to meet the increasing oxygen demand but only up to the point where maximal capacity is reached (Manley 1996). This may explain the higher mean plasma F concentration, Cmax and $\mathrm{AUC}_{(0-90 \mathrm{~min})}$ for the moderate compared to vigorous exercise as participants may have reached their maximum cardiac output when exercising at vigorous intensity. Future studies are therefore needed to include interventions where participants undertake an exercise routine at different intensities (light, moderate and vigorous), for a prolonged period of time.

Urine is the major excretion route for systemically absorbed bioavailable F, with the majority of an ingested $\mathrm{F}$ dose appearing in the urine within the first three hours (Zipkin and Leone, 1957). Our study showed that, on average, $59 \%$ of daily intake of $\mathrm{F}$ was excreted in urine, over a $24 \mathrm{~h}$ period, which is in agreement with the suggested corresponding figure of $60 \%$ for healthy adults (Buzalaf and 
Whitford, 2011). Our study also found that, on average, $21 \%$ of ingested F dose was excreted in urine

299 during the first three hours following F ingestion, corresponding to the value of $20 \%$ reported for healthy adults (Zipkin and Leone, 1957).

301 Our study showed a lower, although not statistically significant, UFE rate over the first $3 \mathrm{~h}$ period for moderate exercise compared with light and vigorous exercise as well as when compared with no exercise (Table 3). However, the UFE rate tended to be higher over the 3-14h period for moderate exercise compared with other exercise intensities including no exercise. These findings indicate that moderate exercise may lead to a delay in urinary F excretion in adults. The lower UFE rate over the first $3 \mathrm{~h}$ period could be explained by the increased production of lactic acid, leading to a more acidic urine and consequently resulting in a higher proportion of ingested $\mathrm{F}$ being reabsorbed (i.e. lower urinary $\mathrm{F}$ excretion). In addition, it is known that a steady-state relationship exists between plasma $\mathrm{F}$ levels and the hydration shell of the bone crystallites (Rao et al., 1995). Thus, another possibility is that moderate exercise increases the absorption of $\mathrm{F}$, thus augmenting plasma $\mathrm{F}$ levels, which in turn would increase F uptake in the hydration shell of the bone crystallites. As plasma F levels start to decrease after the peak is reached, then F present in the hydration shell of the bone crystallites is released back into plasma and excreted in urine over time.

In order to reduce dental caries in children, public health initiatives such as school fluoridated milk programmes have been rolled out across schools in some counties including the UK (Banoczy et al., 2009). However, previous studies have indicated that current UK milk fluoridation programmes do not provide adequate protection for the prevention of dental caries (Ketley and Lennon, 2000). It has been reported that increasing the school milk F dose from $0.5 \mathrm{mg}$ to $0.9 \mathrm{mg}$ per $189 \mathrm{ml}$, in the UK, may still be too low to achieve the World Health Organisation recommended UFE concomitant with optimal F exposure for children aged < 6y (World Health Organization, 2014). In the UK, fluoridated milk, is often provided to schoolchildren during their mid- morning break before undergoing physical activity. In addition, UK children's physical activity levels during break have been reported to be predominantly moderate (Powell et al., 2016). According to our findings, the low UFE observed (Maguire et al., 2013) during monitoring of fluoridated milk programmes may therefore be related to 
the effect of moderate physical activity, that children undertake during their breaks, on F absorption and excretion. F concentrations in blood and urine have been shown not to be influenced by sex (Torra et al., 1998; Del Carmen et al., 2016) However, due to the possible different physiological responses following exercise in children compared to adults, as well as females compared to males, further work is required to determine the effects of exercise on F metabolism in young children and different sexes. These findings can help inform the evidence base for stakeholders and decision makers in dental public health as well as health professionals who may wish to review $\mathrm{F}$ dose and time of administration in different fluoridation programmes.

The main limitations of our study are: (i) the sample size; although the number of participants in this study are comparable with other similar studies in humans (Zohoori et al., 2015) and animals (Whitford et al., 1988; Lombarte et al., 2013); (ii) the F dose; which was based on the optimal F concentration of drinking water of $1 \mathrm{mg} / \mathrm{l}$, and; (iii) the inclusion of only one age group (young adults). Since the peak plasma and bone $\mathrm{F}$ concentrations are directly related to both the age of the individual and $\mathrm{F}$ intakes, any extrapolation of the study findings to other age groups should be made with caution.

Our study also indicated large variation in pharmacokinetic variables between individuals. A study by Ekstrand [Ekstrand, 1978] with a family of five, aged 10 to 38 years old, who ate together and received a water supply with 9.6 ppm F, showed a large variation in plasma F concentration between family members and a much greater within-individual variation during the day (e.g. $40-110 \mathrm{ng} / \mathrm{ml}$ for an adult family member). Some of the relatively wide variation in pharmacokinetic variables between participants might be explained by between-individual differences in physiological variables such as volume and $\mathrm{pH}$ of gastric secretions, gastro-intestinal motility, plasma volume, and urinary $\mathrm{pH}$.

In conclusion, this human experimental study adds to the understanding of the effects of exercise on $\mathrm{F}$ metabolism. The findings suggest that moderate exercise may increase the fraction of ingested $\mathrm{F}$ absorbed systemically and therefore available to produce a biological effect. In addition, moderate exercise may have a tendency to delay the excretion of $\mathrm{F}$ in urine. 
352

Amaral, S.L., Azevedo, L.B., Buzalaf, M.A.R., Fabricio, M.F., Fernandes, M.S., Valentine, R.A., Maguire, A. and Zohoori, F.V. (2018) 'Effect of chronic exercise on fluoride metabolism in fluorosissusceptible mice exposed to high fluoride', Sci Rep, 8(1), p. 3211.

American College of Sports Medicine (2007) ACSM's Health/Fitness Facility Standards and Guidelines, 4E. Human Kinetics.

Banoczy, J., Petersen, P.E. and Rugg-Gunn, A. (eds.) (2009) Milk fluoridation for the prevention of dental caries. Geneva: World Health Organization.

Beaver, W.L., Wasserman, K. and Whipp, B.J. (1986) 'A New Method for Detecting Anaerobic Threshold by Gas-Exchange', J. Appl. Physiol., 60(6), pp. 2020-2027.

Buzalaf, M.A. and Whitford, G.M. (2011) 'Fluoride Metabolism', in Buzalaf, M.A. (ed.) Fluoride and the Oral Environment. Basel, Switzerlan: Karger.

Buzalaf, M.A.R. (2018) 'Review of Fluoride Intake and Appropriateness of Current Guidelines', Adv Dent Res, 29(2), pp. 157-166.

Craig, C.L., Marshall, A.L., Sjostrom, M., Bauman, A.E., Booth, M.L., Ainsworth, B.E., Pratt, M., Ekelund, U., Yngve, A., Sallis, J.F. and Oja, P. (2003) 'International physical activity questionnaire: 12-country reliability and validity', Med Sci Sport Exer, 35(8), pp. 1381-1395.

Evans, C.H. and White, R.D. (2009) Exercise testing for primary care and sports medicine physicians. 1st edn. New York: Springer.

Ketley, C.E. and Lennon, M.A. (2000) 'Urinary fluoride excretion in children drinking fluoridated school milk', Int. J. Paediatr. Dent, 10(4), pp. 260-270.

Lombarte, M., Fina, B.L., Lupo, M., Buzalaf, M.A. and Rigalli, A. (2013) 'Physical exercise ameliorates the toxic effect of fluoride on the insulin-glucose system', J Endocrinol, 218(1), pp. 99103. 
Maguire, A., Walls, R., Steen, N., Teasdale, L., Landes, D., Omid, N., Moynihan, P. and Zohoori, F.V. (2013) 'Urinary Fluoride Excretion in 6- to 7-Year-Olds Ingesting Milk Containing 0.5 or $0.9 \mathrm{mg}$ Fluoride', Caries Res, 47(4), pp. 291-298.

Maguire, A., Zohouri, F.V., Mathers, J.C., Steen, I.N., Hindmarch, P.N. and Moynihan, P.J. (2005) 'Bioavailability of Fluoride in Drinking Water: a Human Experimental Study', J Dent Res, 84(11), pp. 989-993.

Martínez-Mier, E.A., Cury J.A., Heilman J.R., Katz B.P., Levy S.M., Li Y., Maguire A., Margineda J., O’Mullane D., Phantumvanit P., Soto-Rojas A.E., Stookey G.K., Villa A., Wefel J.S., Whelton H. , Whitford G.M., Zero D.T., Zhang W. and Zohouri, V. (2011) 'Development of gold standard ionselective electrode-based methods for fluoride analysis ', Caries Res, 45, pp. 3-12.

Neufer, P.D. (1989) 'The Effect of Detraining and Reduced Training on the Physiological Adaptations to Aerobic Exercise Training', Sport Med, 8(5), pp. 302-320.

Noble, B.J., Borg, G.A.V., Jacobs, I., Ceci, R. and Kaiser, P. (1983) 'A Category-Ratio Perceived Exertion Scale - Relationship to Blood and Muscle Lactates and Heart-Rate', Med Sci Sport Exer, 15(6), pp. 523-528.

Palmer, C. and Wolfe, S.H. (2005) 'Position of the American Dietetic Association: the impact of fluoride on health', J Am Diet Assoc, 105(10), pp. 1620-8.

Powell, E., Woodfield, L.A. and Nevill, A.A.M. (2016) 'Children's physical activity levels during primary school break times: A quantitative and qualitative research design', Eur. Phys. Educ. Rev., 22(1), pp. 82-98.

Rao, H.V., Beliles, R.P., Whitford, G.M. and Turner, C.H. (1995) 'A physiologically based pharmacokinetic model for fluoride uptake by bone', Regulatory Toxicology and Pharmacology, 22(1), pp. 30-42.

Schwab, P. and Scalapino, K. (2011) 'Exercise for bone health: rationale and prescription', Curr Opin Rheumatol, 23(2), pp. 137-41. 
Taves, D.R. (1968) 'Separation of fluoride by rapid diffusion using hexamethyldisiloxane', Talanta,

401

402

403

404

405

406

407

408

409

410

411

412

413

414

415

416

417

418

419

420

421

422

423 15(9), pp. 969-974.

ten Cate, J.M. and Buzalaf, M.A.R. (2019) 'Fluoride Mode of Action: Once There Was an Observant Dentist ', J Dent Res, 98(7), pp. 725-730.

Torra, M., Rodamilans, M. and J Corbella,J. (1998) Serum and urine fluoride concentration: Relationships to age, sex and renal function in a non-fluoridated population, Sci Total Environ, 220 (1), 81-5. doi.org/10.1016/S0048-9697(98)00248-4.

Del Carmen, A.F., Javier, F.H., Aline, C.C. (2016)Dental fluorosis, fluoride in urine, and nutritional status in adolescent students living in the rural areas of Guanajuato, Mexico. J Int Soc Prev Community Dent. 6(6):517-522. doi:10.4103/2231-0762.195510

Whitford, G.M. (1996) The Metabolism and Toxicity of Fluoride. Basel: Karger.

Whitford, G.M., Birdsong-Whitford, N.L. and Lowe, S.R. (1988) 'Fluoride pharmacokinetics: effect of light exercise in rats.', Caries Res, 22, p. Abs 106.

Willems, H.M.E., van den Heuvel, E.G.H.M., Schoemaker, R.J.W., Klein-Nulend, J. and Bakker, A.D. (2017) 'Diet and Exercise: a Match Made in Bone', Current Osteoporosis Reports, 15(6), pp. $555-563$.

World Health Organization (2014) Basic methods for assessing renal fluoride excretion in community prevention programmes for oral health Geneva, Switzerland: World Health Organization,.

Yu, H., Jiang, N., Yu, X., Zhao, Z., Zhang, X. and Xu, H. (2018) 'The role of TGFbeta receptor 1smad3 signaling in regulating the osteoclastic mode affected by fluoride', Toxicology, 393, pp. 73-82.

Zipkin, I. and Leone, N.C. (1957) 'Rate of urinary fluoride output in normal adults', Am J Public Health, 47(7), pp. 848-51.

Zohoori, F.V., Innerd, A., Azevedo, L.B., Whitford, G.M. and Maguire, A. (2015) 'Effect of exercise on fluoride metabolism in adult humans: a pilot study', Sci Rep, 5. 
427 The authors declare no potential conflicts of interest with respect to the authorship and/or publication 428 of this article.

429 Author contributions:

430 FVZ and MM conceived the study; FVZ, MM, and LBA designed the study; MM collected and 431 analyzed the samples; FVZ supervised the project with help from LBA; FVZ, and MM analyzed the 432 data and LBA, AM, and MB contributed to the interpretation of the results; FVZ, MM and AM took 433 the lead in writing the manuscript. All authors read, provided critical feedback and approved the 434 submitted paper.

435 Acknowledgement

436 This study was supported by an internal grant from Teesside University as well as an external grant 437 from The Borrow Foundation. 
Table 1. Mean (SD) plasma fluoride (F) concentrations (ngF/ml), during experimental sessions; no exercise (control), light, moderate and vigorous exercise.

441

\begin{tabular}{|l|c|c|c|c|}
\hline \multirow{2}{*}{$\begin{array}{l}\text { Post-F ingestion } \\
\text { plasma collection }\end{array}$} & \multicolumn{4}{|c|}{ Exercise intensity } \\
\cline { 2 - 5 } time & No exercise & Light & Moderate & Vigorous \\
& (Control) & & & \\
\hline 30 minutes (T1) & $20.4(14.7)$ & $150.9(54.9)$ & $241.3(130.1)$ & $93.8(36.7)$ \\
\hline 45 minutes (T2) & $29.6(16.6)$ & $147.9(77.5)$ & $263.1(113.6)$ & $136.9(59.8)$ \\
\hline 60 minutes (T3) & $33.6(26.9)$ & $134.6(104.7)$ & $238.7(74.1)$ & $127.5(24.9)$ \\
\hline 90 minutes (T4) & $16.6(12.5)$ & $110.0(98.7)$ & $205.2(83.3)$ & $111.4(42.9)$ \\
\hline
\end{tabular}

442

443 
444 Table 2. Mean (SD) pharmacokinetic parameters for plasma following ingestion of fluoride (F) tablet $445 \quad(1.0 \mathrm{mg} \mathrm{F})$ by exercise intensity.

446

\begin{tabular}{|l|c|c|c|c|}
\hline \multirow{2}{*}{$\begin{array}{l}\text { Pharmacokinetic parameters } \\
\text { for plasma F }\end{array}$} & \multicolumn{4}{|c|}{ Exercise intensity } \\
\cline { 2 - 5 } & $\begin{array}{c}\text { No exercise } \\
(\text { Control })\end{array}$ & Light & Moderate & Vigorous \\
\hline $\mathrm{T}_{\max }(\mathrm{min})^{\mathrm{a}}$ & $50.3(11.0)$ & $42.9(13.5)$ & $45.9(10.5)$ & $51.3(10.9)$ \\
\hline $\mathrm{C}_{\max }(\mathrm{ngF} / \mathrm{ml})^{\mathrm{b}}$ & $27.0(24.3)$ & $105.6(41.7)$ & $226.2(115.6)$ & $94.2(58.1)$ \\
\hline AUC $_{(0-90 \min )}\left(\mathrm{ngF} \cdot \min . \mathrm{ml}^{-1}\right)^{\mathrm{c}}$ & $1474(939)$ & $6920(3506)$ & $15058(6596)$ & $5542(2264)$ \\
\hline
\end{tabular}

447

448 a) Lag time to maximum F concentration

449 b) Maximum F concentration

450

c) Area under the curve

451 
Table 3. Mean (SD) urinary fluoride excretion (UFE; $\mu \mathrm{gF}$ ) for different time-controlled periods 453 during experimental sessions for no exercise (control), light, moderate and vigorous exercise.

\begin{tabular}{|l|c|c|c|c|}
\hline \multirow{2}{*}{ UFE (Time period) } & \multicolumn{3}{|c|}{ Exercise intensity } \\
\cline { 2 - 5 } & No exercise & Light & Moderate & Vigorous \\
& Control) & & & \\
\hline UFE $_{0-3 \mathrm{~h}}(9: 00-12: 00)$ & $302.7(354.4)$ & $222.3(64.0)$ & $119.4(132.4)$ & $213.5(100.0)$ \\
\hline UFE $_{3-8 \mathrm{~h}}(12: 00-17: 00)$ & $214.0(167.8)$ & $207.4(116.4)$ & $242.9(100.3)$ & $130.8(74.1)$ \\
\hline UFE $_{8-14 h}(17: 00-23: 00)$ & $130.3(122.2)$ & $196.0(206.7)$ & $207.8(134.7)$ & $124.5(52.5)$ \\
\hline UFE $_{0-14}(09: 00-23: 00)$ & $638.8(565.5)$ & $718.7(296.8)$ & $574.6(281.1)$ & $450.5(206.1)$ \\
\hline
\end{tabular}

455

456

457

458

459

460

461

462

463 

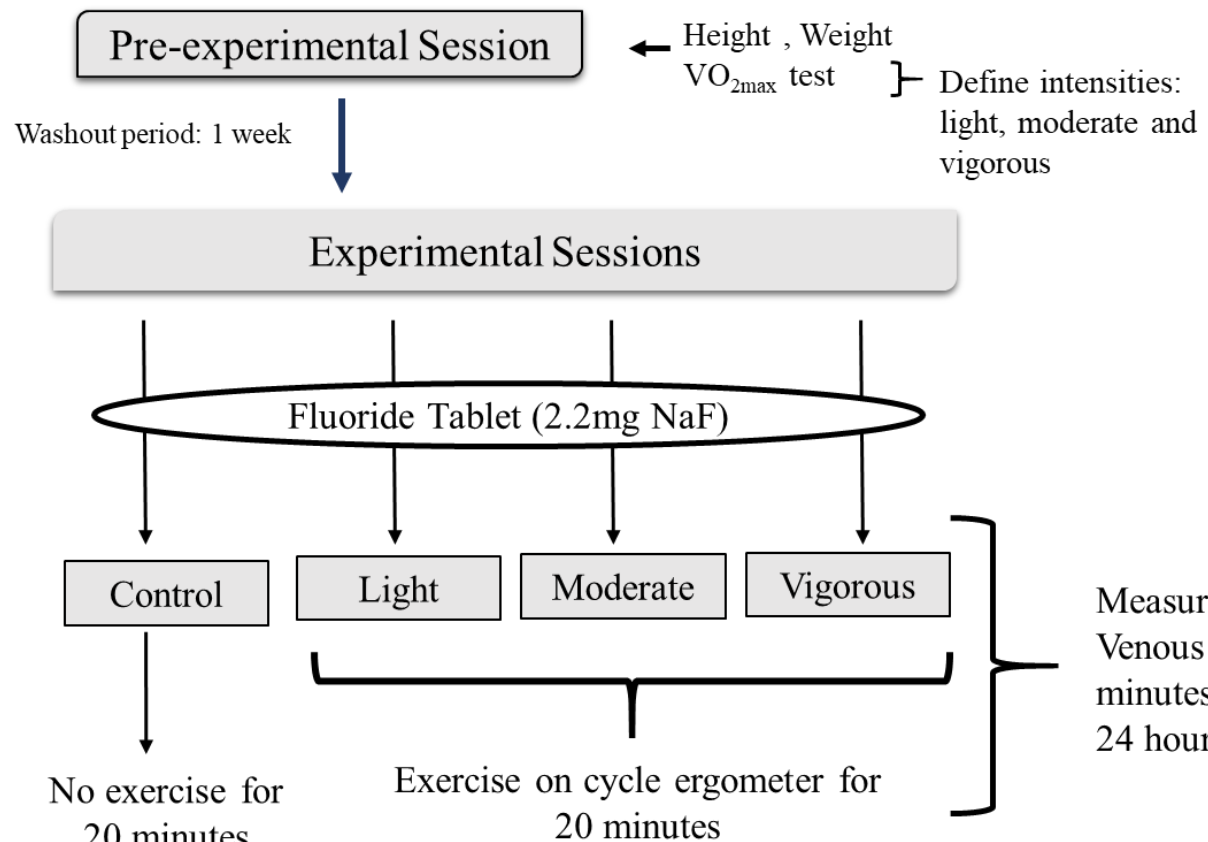

Measures:

Venous blood at $0,30,45,60 \& 90$ minutes post fluoride ingestion 24 hour pooled urine at intervals 20 minutes

Figure 1. Experimental procedure and sample collection 
472

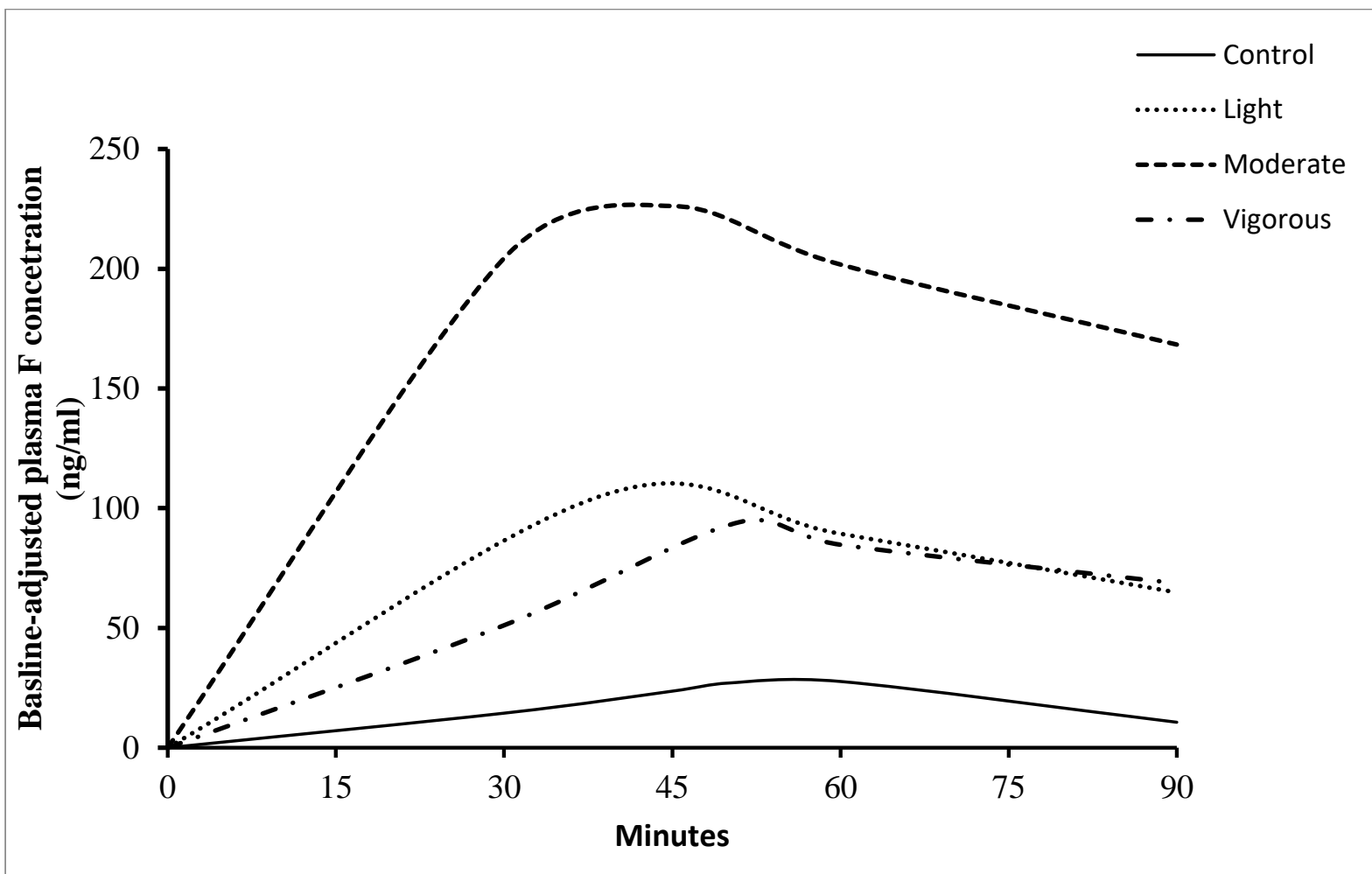

473

474 Figure 2. Baseline-adjusted plasma $\mathrm{F}$ concentration $(\mathrm{ng} / \mathrm{ml})$ over the $0-90$ minute post $\mathrm{F}$ ingestion 475 period.

476

477 


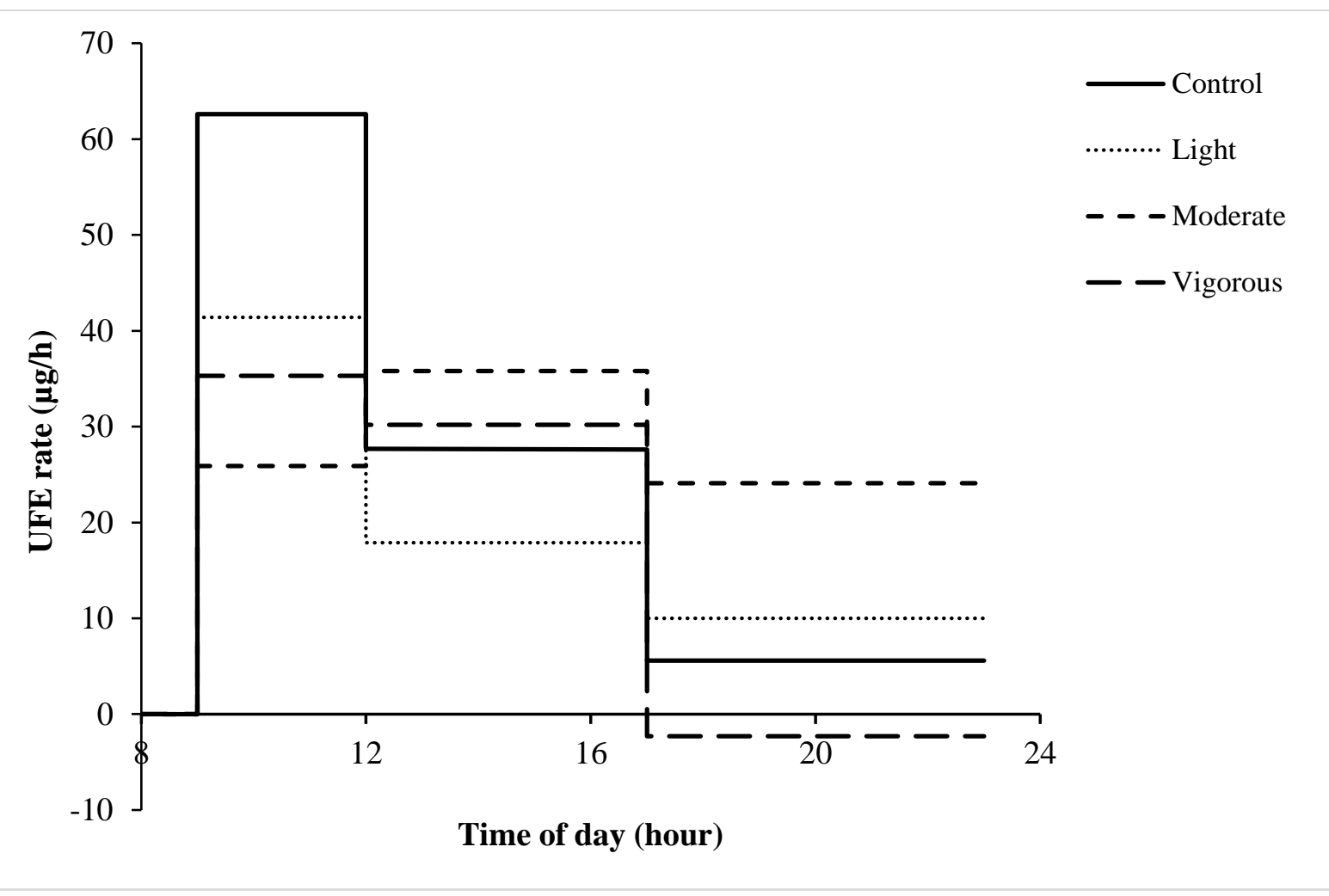

480

481 Figure 3. Mean baseline-adjusted UFE rate $(\mu \mathrm{gF} / \mathrm{h})$ across the 4 time-controlled periods of collection according to exercise intensity; no exercise (control (blue line)), light (green line), moderate (brown line) and vigorous exercise (yellow line) 